\title{
Throughput Analysis of Type-I HARQ Strategies in Two-Way Relay Channels
}

\author{
Fabio Iannello and Osvaldo Simeone \\ CWCSPR, New Jersey Institute of Technology \\ Newark, New Jersey 07102-1982, USA \\ Email: \{fabio.iannello, osvaldo.simeone\}@njit.edu
}

\begin{abstract}
A Two-Way Relay Channel (TWRC) is studied over quasi-static fading channels by focusing on the throughput of Type-I HARQ strategies. An upper bound is evaluated, along with a number of achievable results obtained by proposing different protocols combining Type-I HARQ with standard amplify or decode-and-forward techniques or more sophisticated lattice code-based strategies. Performance comparison among the different protocols shows a trade-off between the achievable throughput and the system overhead required to obtain partial channel state information at the nodes.
\end{abstract}

\section{INTRODUCTION}

In recent years, the study of the Two-Way Relay channel (TWRC) has illuminated the possibility of designing transmission strategies that exploit in novel ways the inherent properties of broadcasting and superposition of the wireless medium. In a TWRC (see Fig. 1) two terminals communicate with each other via a relay. In essence, in a TWRC, the relay can leverage the fact that the signals transmitted by the terminals superimpose "on air", to broadcast back to the terminals some function of such "combined" signal. It is then up to the terminals to sort out the signal of interest from the superposition by using knowledge of the local transmitted codeword (side information) [1]-[3]. Most previous work on the TWRC has focused on the case of fixed (deterministic) channels, and has culminated in the recent works [4] and [5], where the cut-set upper bound conjectured in [2] was closely approached by techniques based on structured (lattice) codes. Other possible strategies to be employed at the relay include amplify-and-forward (AF) [1] and decode-and-forward (DF) [3].

Departing from most previous analysis, in this work we focus on the performance in terms of average throughput of TWRCs over quasi-static fading channels. We consider applications where reliable packet delivery is guaranteed by a Type-I HARQ retransmission policy. In the previous work [1], the authors analyze the performance of Type-I ARQ protocols assuming uncoded transmission (i.e., ARQ rather than HARQ, see also [6]). Here instead we cast the throughput analysis in an information-theoretic framework, assuming capacityachieving codes and HARQ. We further extend the analysis of [1] (on DF-based protocols), considering joint decoding capability at the relay and the use of structured codes. Specifically, an upper bound on the throughput is evaluated, along with a number of achievable results obtained by proposing different

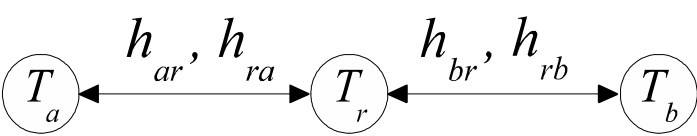

Fig. 1. The Two-Way Relay Channel.

protocols that combine Type-I HARQ with standard AF or DF techniques or more sophisticated lattice code-based strategies. Performance comparison among the different protocols shows a trade-off between the achievable throughput and the system overhead required to obtain partial channel state information at the nodes.

\section{SYSTEM MODEL}

We consider a TWRC with three half-duplex nodes. Terminals $T_{a}$ and $T_{b}$ (in the sequel also called users) want to establish a bidirectional communication between each other, with the help of the terminal $T_{r}$, which acts as a relay and has no message of its own to transmit (see Fig. 1). It is assumed that the direct link between $T_{a}$ and $T_{b}$ is not available. Each user has an infinite backlog of data intended for the other user, and time is slotted, with slots of $n$ channel uses. At each slot in which terminal $T_{a}\left(T_{b}\right)$ is polled by the relay for transmission, $T_{a}\left(T_{b}\right)$ extracts $n R_{a}\left(n R_{b}\right)$ bits, denoted as $W_{a}\left(W_{b}\right)$, from the backlog. The transmission rate (bits/channel use or bps/Hz assuming no bandwidth expansion) of terminal $T_{a}\left(T_{b}\right)$ is denoted by $R_{a}\left(R_{b}\right)$ and it is assumed to be fixed. The encoding process takes place by mapping the current message $W_{a}\left(W_{b}\right)$ onto a $(n \times 1)$ codeword $\mathbf{x}_{a}\left(W_{a}\right)\left(\mathbf{x}_{b}\left(W_{b}\right)\right)$, selected from a codebook of size $2^{n R_{a}}\left(2^{n R_{b}}\right)$. The power constraint is given by (we drop the dependence on $W_{i}$ for simplicity of notation) $\frac{1}{n} \mathbf{x}_{i}^{H} \mathbf{x}_{i} \leq P_{i}$ for $i \in\{a, b, r\}$. Reception at each node is subject to Additive White Gaussian Noise (AWGN), represented by the complex $(n \times 1)$ vector $\mathbf{n}_{i} \sim \mathcal{C N}(0, \mathbf{I})$. The channel $h_{i j}$ between nodes $i$ and $j$, is assumed to be frequency flat and constant over the entire time slot, and is characterized by Rayleigh fading, $\left|h_{i j}\right|^{2} \sim \exp (1)$. In time slots where one or both users transmit (uplink) the signal received at the relay node is given by:

$$
\mathbf{y}_{r}=h_{a r} \mathbf{x}_{a}+h_{b r} \mathbf{x}_{b}+\mathbf{n}_{r},
$$

with the convention that if only one terminal $T_{a}$ or $T_{b}$ transmits, either $\mathbf{x}_{b}$ or $\mathbf{x}_{a}$ is equal to the $(n \times 1)$ null vector 
$\mathbf{0}_{n}$. For transmission in downlink time slots, the relay creates its own codeword $\mathbf{x}_{r}$ as a function of the signals received in the previous time slots. The signal received at terminal $T_{i}$ is:

$$
\mathbf{y}_{i}=h_{r i} \mathbf{x}_{r}+\mathbf{n}_{i}
$$

with $i \in\{a, b\}$. Different assumptions on the knowledge of the channel gains at the nodes will be discussed below for the considered protocols. In all cases, the current fading conditions in any slot may not support transmissions at fixed rates causing receivers to be in outage. As described below, outage events are dealt with via retransmissions (HARQ).

\section{THROUGHPUT}

In this paper, we focus on data applications in which reliable (zero-error) transmission is required while latency is not an issue. For this reason, we resort to Type-I HARQ strategies, so that copies of erroneously received packets are retransmitted while the receivers discard the previous copies and decodes based only on the current retransmissions. Similarly, it is assumed that the relay may construct the transmitted signal $\mathbf{x}_{r}$ only based on the current (re)transmissions of either user (i.e., no combination of packets of the same user is allowed). We evaluate this performance by measuring the long-term throughput defined as:

$$
\eta=\lim _{M \rightarrow \infty} \frac{1}{M} \sum_{m=1}^{M} R_{a} I_{a}[m]+R_{b} I_{b}[m],
$$

where $I_{i}[m]$, for $i \in\{a, b\}$, is an indicator function of a successful decoding event, defined as:

$$
I_{i}[m]: T_{j} \text { decodes a packet from } T_{i} \text { in time slot } m,
$$

for $i \neq j$, with $j \in\{a, b\}$. Whenever the considered protocols satisfy appropriate assumptions of stationary and ergodicity, one may substitute (3) with the ensemble average throughput:

$$
\eta=R_{a} \bar{I}_{a}+R_{b} \bar{I}_{b}
$$

where $\bar{I}_{i}=E\left[I_{i}[m]\right]$ and $E[\cdot]$ denotes the expectation over fading, while the dependence on the time slot has been dropped by the assumption of stationarity. Finally, we assume that the ACK and NACK messages, used to coordinate the terminals, are always correctly received and their length is negligible compared to the length of the data message.

1) Upper Bound: A simple upper bound to the average throughput (5) with Type-I HARQ can be obtained, for given transmission rates $\left(R_{a}, R_{b}\right)$, by assuming that the two terminals can transmit without interference from the other user. In this case the optimal operation at the relay is clearly DF (the final destination cannot decode anything that cannot be decoded at the relay) and standard i.i.d. "Gaussian codebooks" are optimal. As such, any terminal, say $T_{a}$, sees a two-hop link towards $T_{b}$ whose maximum throughput using Type-I HARQ can be calculated as ${ }^{1}$ :

$$
\tilde{\eta}_{a}=R_{a} \frac{\left(1-p_{\text {out }, a r}\right)\left(1-p_{\text {out }, r b}\right)}{\left(2-p_{\text {out }, a r}-p_{\text {out }, r b}\right)}
$$

where $p_{\text {out }, i j}$ is the outage probability on the link between $T_{i}$ and $T_{j}$ which is given by (recall the assumption of "Gaussian codebooks" and Rayleigh fading [8]):

$$
p_{\text {out }, i j}=\operatorname{Pr}\left\{R_{i}>C\left(P_{i}\left|h_{i j}\right|^{2}\right)\right\}=1-e^{\left(-\frac{2^{R_{i}-1}}{P_{i}}\right)},
$$

where $C(x)=\log _{2}(1+x)$. Similarly an upper bound $\tilde{\eta}_{b}$ can be obtained for $T_{b}$. Thereby, we can obtain the upper bound for the throughput as $\eta \leq \eta_{U B}=\tilde{\eta}_{a}+\tilde{\eta}_{b}$.

\section{AF-BASED SCHEME}

We consider at first an AF-based scheme that was first proposed in [1] for uncoded transmission. The AF-based scheme exploits the inherent additive property of the wireless channel to combine the users' signals. Here, the terminals $T_{a}$ and $T_{b}$ always transmit simultaneously to the relay, while the latter amplifies and broadcasts the received signal choosing an amplification factor respecting its power constraint $P_{r}$. For this, the relay needs to compute the power of the received signal $\left|h_{a r}\right|^{2} P_{a}+\left|h_{b r}\right|^{2} P_{b}+1$ (see (1)), which can be estimated as $\frac{1}{n} \mathbf{y}_{r}^{H} \mathbf{y}_{r}$, but it does not need to know explicitly the channel gains, while terminal $T_{a}\left(T_{b}\right)$ needs to know the products $h_{a r} h_{r a}$ and $h_{a r} h_{b r}\left(h_{b r} h_{r b}\right.$ and $\left.h_{b r} h_{a r}\right)$ for decoding (see below). It is finally noted that all the achievable schemes derived throughout the paper employ standard i.i.d. "Gaussian codebooks".

\section{A. Protocol}

The protocol adopted for the AF-based scheme is [1]:

- In odd time slots, terminals $T_{a}$ and $T_{b}$ transmit simultaneously their respective codewords, $\mathbf{x}_{a}$ and $\mathbf{x}_{b}$, which can be retransmissions of the previous messages.

- In even time slots, the relay amplifies and broadcasts the signal received in the previous odd slot as $\mathbf{x}_{r}=$ $\sqrt{G} \mathbf{y}_{r}$, with $G=n P_{r} / \mathbf{y}_{r}^{H} \mathbf{y}_{r}$. Terminal $T_{a}$ receives $\mathbf{y}_{a}=h_{r a} \sqrt{G}\left(h_{a r} \mathbf{x}_{a}+h_{b r} \mathbf{x}_{b}+\mathbf{n}_{r}\right)+\mathbf{n}_{i}$, subtracts off its weighted transmitted signal $\left(\sqrt{G} h_{r a} h_{a r} \mathbf{x}_{a}\right)$ and decodes, and similarly for terminal $T_{b}$. Depending whether $T_{a}$ $\left(T_{b}\right)$ has correctly decoded $W_{b}\left(W_{a}\right)$ or not, $T_{b}\left(T_{a}\right)$ will respectively transmit either a new or an old message (Type-I HARQ) in the next odd slot.

\section{B. Throughput}

With the AF-based scheme protocol above, successful transmission can take place only during even time slots, so that the indicator variables $\bar{I}_{i}$ are given by $\bar{I}_{i}^{(A F)}=$ $\frac{1}{2}\left(1-p_{\text {out }, j}\right)$, with $i, j \in\{a, b\}$ and $i \neq j$. Factor $1 / 2$ comes from the equal portion of even/odd time slots, and

\footnotetext{
${ }^{1}$ The average number of slots necessary for a correct transmission from $T_{a}$ to $T_{r}$ is given by $N_{U L}=1 /\left(1-p_{\text {out }, a r}\right)$. Similarly from $T_{r}$ to $T_{b}$ $N_{D L}=1 /\left(1-p_{\text {out }, r b}\right)$. The total average number of slots is therefore: $N_{t o t}=N_{U L}+N_{D L}$ and the throughput is given by $\tilde{\eta}_{a}=R_{a}\left(N_{t o t}\right)^{-1}$.
} 
$p_{\text {out }, j}=\operatorname{Pr}\left\{R_{i}>C\left(\frac{G\left|h_{r j}\right|^{2}\left|h_{i r}\right|^{2} P_{i}}{G\left|h_{r j}\right|^{2}+1}\right)\right\}$ is the probability that the terminal $T_{j}$ does not decode the message of terminal $T_{i}$ (see [7] for further details on $p_{\text {out }, j}$ ). Substituting $\bar{I}_{a}^{(A F)}$ and $\bar{I}_{b}^{(A F)}$ in (5) we obtain (see also [1]):

$$
\eta_{A F}=\frac{1}{2}\left[R_{a}\left(1-p_{\text {out }, b}\right)+R_{b}\left(1-p_{\text {out }, a}\right)\right] .
$$

\section{DF-BASED SCHEMES}

In this section, we consider two DF-based protocols. In these schemes, the relay first decodes the users' messages $W_{a}$ and $W_{b}$, and then creates its own codeword $\mathbf{x}_{r}\left(W_{a}, W_{b}\right)$ for downlink transmission. Codeword $\mathbf{x}_{r}$ is obtained from a "Gaussian codebook" of $2^{n\left(R_{a}+R_{b}\right)}$ codewords. We refer to these schemes as single and joint decoding-DF (SDF and JDF) protocols. In the former one, previously proposed and analyzed for uncoded transmission in [1], the relay performs single-user detection, while in the latter protocol joint decoding (multiuser detection) is carried out at the relay. Since the relay needs to decode users' messages it requires the knowledge of the channel gains $h_{a r}$ and $h_{b r}$, whereas node $T_{a}\left(T_{b}\right)$ needs to know only $h_{r a}\left(h_{r b}\right)$.

\section{A. SDF-based Protocol}

For the throughput analysis of the SDF-based type-I HARQ scheme, the system can be studied as a Markov chain with states given by the status of the relay's buffer $B_{r}$, as proposed in [1] (see Fig. 2). This buffer can assume the following states: 1) $S_{0}: B_{r}$ does not contain any messages. 2) $S_{a}: B_{r}$ contains $T_{a}$ 's message, say $W_{a}$ (of $n R_{a}$ bits), intended for $T_{b}$. 3) $S_{b}$ : $B_{r}$ contains $T_{b}$ 's message, say $W_{b}$ (of $n R_{b}$ bits), intended for $T_{a}$. 4) $S_{a b}: B_{r}$ contains $T_{a}$ and $T_{b}$ 's messages intended respectively for $T_{b}$ and $T_{a}$. Based on these states we propose the following HARQ protocol:

- $S_{0}$ : when $B_{r}=\{\varnothing\}, T_{r}$ keeps on polling $T_{a}$ until a new message $W_{a}$ is correctly received.

- $S_{a}$ : when $B_{r}=\left\{W_{a}\right\}, T_{r}$ keeps on polling $T_{b}$ until a new message $W_{b}$ is correctly received.

- $S_{b}$ : when $B_{r}=\left\{W_{b}\right\}, T_{r}$ keeps on polling $T_{a}$ until a new message $W_{a}$ is correctly received

- $S_{a b}$ : when $B_{r}=\left\{W_{a}, W_{b}\right\}, T_{r}$ creates and broadcasts its own codeword $\mathbf{x}_{r}\left(W_{a}, W_{b}\right)$ selected from a codebook of size $2^{n\left(R_{a}+R_{b}\right)}$. The destination terminal $T_{a}\left(T_{b}\right)$ knows its own message $W_{a}\left(W_{b}\right)$ and thus can decode $W_{b}\left(W_{a}\right)$ from the received signal $\mathbf{y}_{a}\left(\mathbf{y}_{b}\right)$ seeking in a codebook $\mathbf{x}\left(W_{a}, W_{b}\right)$ of size $2^{n R_{b}}\left(2^{n R_{a}}\right)$.

1) SDF-based throughput: Successful transmission for terminal $T_{a}$ can occur only when there is a transition from state $S_{a b}$ to $S_{a}$ or $S_{0}$, and similarly for terminal $T_{b}$ when there is a transition from $S_{a b}$ to $S_{b}$ or $S_{0}$. The indicator variable $\bar{I}_{i}$ can be obtained as $\bar{I}_{i}^{(S D F)}=\pi_{a b}^{(S D F)}\left(1-p_{\text {out }, r j}\right)$, with $i, j \in\{a, b\}$, for $i \neq j$, where $p_{\text {out }, i j}$ is (7), and $\pi_{a b}^{(S D F)}$ is the probability that the relay's buffer is in the $S_{a b}$ state, which can be obtained, using standard techniques, by finding the steadystate distribution of the Markov chain in Fig. 2. Substituting

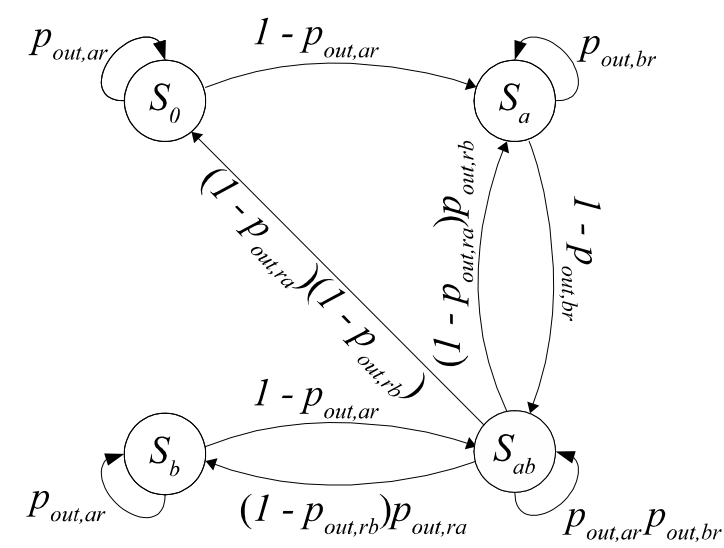

Fig. 2. Markov chain for the Single decoding-DF (SDF) scheme.

$$
\begin{aligned}
& \bar{I}_{a}^{(S D F)} \text { and } \bar{I}_{b}^{(S D F)} \text { in (5) we obtain: } \\
& \eta_{S D F}=\pi_{a b}^{(S D F)}\left[R_{a}\left(1-p_{\text {out }, r b}\right)+R_{b}\left(1-p_{\text {out }, r a}\right)\right] .
\end{aligned}
$$

In the case of a fully symmetric TWRC, $R_{a}=R_{b} \triangleq R$, $P_{a}=P_{b}=P_{r} \triangleq P$, we have $p_{\text {out }, r a}=p_{\text {out }, r b} \triangleq p_{\text {out }}$, and we can find that $\pi_{a b}^{(S D F)}=1 / 3$ and therefore the throughput is given by $\eta_{S D F}=\frac{2}{3} R\left(1-p_{\text {out }}\right)$. Moreover, the transmission rate $R^{*}$ maximizing the latter throughput for a given SNR $P$ can be found analytically [9] as $R^{*}(P)=W_{0}(P)$, where $W_{0}(\cdot)$ is the Lambert $\mathrm{W}$ function main branch.

\section{B. JDF-based Protocol}

In this section we introduce a JDF-based type-I HARQ scheme where users are allowed to transmit their signals simultaneously. As described in section V-A the relay's buffer can assume only four states, based on which we can propose to implement the following protocol:

- $S_{0}$ : when $B_{r}=\{\varnothing\}, T_{r}$ polls $T_{a}$ and $T_{b}$ to get new messages, say $W_{a}$ (of $n R_{a}$ bits) and $W_{b}$ (of $n R_{b}$ bits). Here the relay always performs joint decoding.

- $S_{a}$ : when $B_{r}=\left\{W_{a}\right\}, T_{r}$ keeps on polling $T_{b}$ until a new message $W_{b}$ is correctly received.

- $S_{b}$ : when $B_{r}=\left\{W_{b}\right\}, T_{r}$ keeps on polling $T_{a}$ until a new message $W_{a}$ is correctly received.

- $S_{a b}$ : when $B_{r}=\left\{W_{a}, W_{b}\right\}, T_{r}$ creates and broadcasts its own codeword $\mathbf{x}_{r}\left(W_{a}, W_{b}\right)$ selected from a codebook of size $2^{n\left(R_{a}+R_{b}\right)}$. The terminals can decode the messages from the received signal as described in section V-A.

1) Throughput: For JDF-based scheme, we need to consider the Markov chain shown in Fig. 3. Here, the uplink transition probabilities from $S_{0}$ are related to the performance of joint decoding at the relay. During joint decoding, the relay can respectively decode both $W_{a}$ and $W_{b}$ with probability $p_{\text {joint }}^{J}$; only $W_{a}\left(W_{b}\right)$ with probability $p_{\text {out }, b}^{J}\left(p_{\text {out }, a}^{J}\right)$; neither $W_{a}$ nor $W_{b}$ with probability $p_{\text {out,comm }}^{J}$. These probabilities, related by $p_{\text {joint }}^{J}=1-p_{\text {out }, a}^{J}-p_{\text {out }, b}^{J}-p_{\text {out }, \text { comm }}^{J}$, have been derived in [8] for Rayleigh fading and they are defined as:

$$
p_{\text {out }, j}^{J}=\operatorname{Pr}\left\{R_{j}>C\left(P_{j}\left|h_{j r}\right|^{2}\right), R_{i} \leq C\left(\frac{P_{i}\left|h_{i r}\right|^{2}}{1+P_{j}\left|h_{j r}\right|^{2}}\right)\right\},
$$




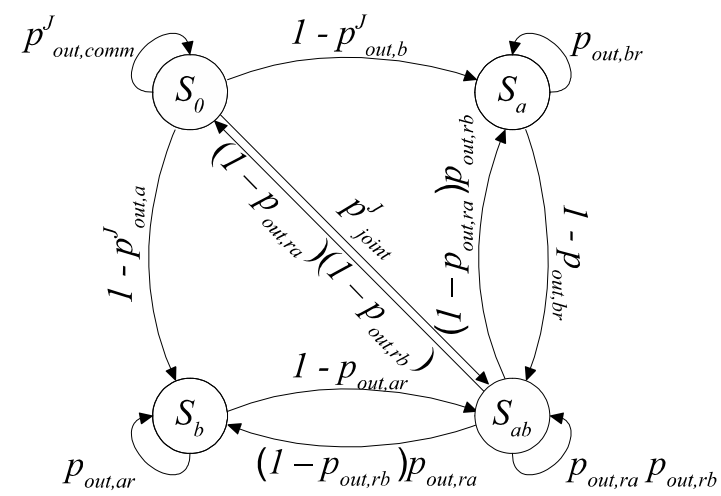

Fig. 3. Markov chain for the Joint decoding - DF (JDF)-based scheme.

with $i, j \in\{a, b\}$, for $i \neq j$, and:

$$
\begin{aligned}
p_{\text {out }, \text { comm }}^{J}=\operatorname{Pr}\{ & R_{a}>C\left(\frac{P_{a}\left|h_{a r}\right|^{2}}{1+P_{b}\left|h_{b r}\right|^{2}}\right), \\
& R_{b}>C\left(\frac{P_{b}\left|h_{b r}\right|^{2}}{1+P_{a}\left|h_{a r}\right|^{2}}\right), \\
& \left.R_{a}+R_{b}>C\left(P_{a}\left|h_{a r}\right|^{2}+P_{b}\left|h_{b r}\right|^{2}\right)\right\}
\end{aligned}
$$

The throughput is only determined by the transitions originating from state $S_{a b}$, so that we have (recall (9)):

$$
\eta_{J D F}=\pi_{a b}^{(J D F)}\left[R_{a}\left(1-p_{\text {out }, r b}\right)+R_{b}\left(1-p_{\text {out }, r a}\right)\right],
$$

where $p_{o u t, r j}$, with $j \in\{a, b\}$, is given by (7).

\section{Vi. Structured Codes}

Finally, in this section, we introduce a type-I HARQ scheme based on the use of structured codes for uplink and downlink transmission as proposed in [5] (see also [4]). The basic idea behind the use of these codes is the fact that the relay does not need to know the users' messages (as enforced by DF), but only needs to broadcast sufficient information so that the terminals are able to decode. Thanks to the group structure of the lattice codes used by the terminals, the combination of two codewords $\mathbf{x}_{a}$ and $\mathbf{x}_{b}$ received at the relay is still a codeword of the original codebook, so that the relay can decode such combination (rather than the individual codewords). The philosophy is similar to that of AF schemes, but here "denoising" is also performed at the relay via decoding of the "combined" codeword. The relay then broadcasts the "combined" codeword; and the users are able to subtract off their self-component and decode the other message thanks to the available side information. Each user, say $T_{a}$, is assumed to know $h_{a r}, h_{r a}$ and the amplitude $\left|h_{b r}\right|$, while the relay only needs $\left|h_{a r}\right|$ and $\left|h_{b r}\right|$ (see below).

\section{A. Review of the Lattice scheme [5]}

In this section we briefly review the lattice-based scheme proposed in [5] for $h_{a r}=1$ and $h_{r a}=1$. The codebook $\mathcal{C}_{a}$ used by terminal $T_{a}$ is obtained from a lattice $\Lambda$, and has a second moment per dimension, say $P_{a} \geq P_{b}$. The codebook $\mathcal{C}_{b}$ for the terminal $T_{b}$ can be obtained by removing the codewords with the largest power from $\mathcal{C}_{a}$ until its average

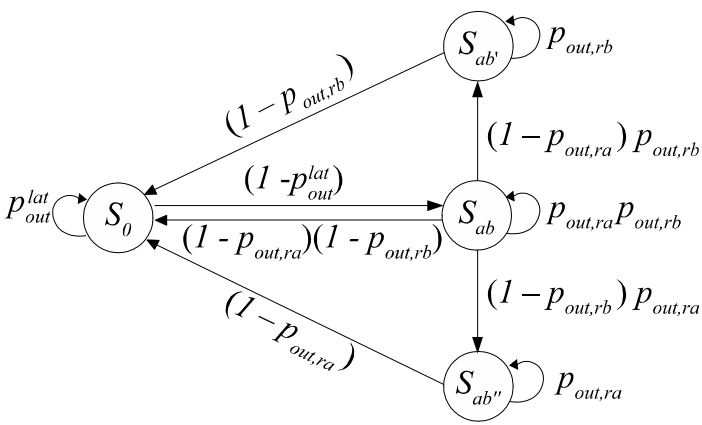

Fig. 4. Markov chain for the lattice code-based scheme.

power is less than or equal to $P_{b}$. Terminal $T_{a}\left(T_{b}\right)$ maps its message $W_{a}\left(W_{b}\right)$ onto a codeword $\mathbf{x}_{a}\left(\mathbf{x}_{b}\right)$ after adding a convenient dither vector. In the absence of fading, it is shown in [5] that the relay can decode the sum of the two users' codewords even if the users' transmitted powers are different (while in [4] authors consider $P_{a}=P_{b}$ ). Here in the presence of fading, terminals are required to pre-compensate the effect of the uplink channel phase, so that the signal received at the relay is $\mathbf{y}_{r}=\left|h_{a r}\right| \mathbf{x}_{a}+\left|h_{b r}\right| \mathbf{x}_{b}+\mathbf{n}_{r}$. The terminals' codebooks must then be chosen (as in [5]) by considering the effective users' powers at the relay $\left(P_{a}\left|h_{a r}\right|\right.$ and $\left.P_{a}\left|h_{b r}\right|\right)$. It is noted that due to the channel state information, the outage in uplink becomes a predictable event, and in practice the users will not transmit in such time-slots (see description of the protocol below). It is finally remarked that an alternative implementation of a lattice-based scheme may be possible even in the presence of unknown channel gains, by exploiting the compute-and-forward strategy of [10]. This is the subject of ongoing research.

\section{B. Lattice-based Protocol}

With lattice codes, unlike JDF, in uplink we can have either correct decoding of the compound codeword or decoding error, that is, single codewords cannot be retrieved due to the utilization of structured codes. Thus the relay's buffer can assume only two states, depending whether or not the "combined" message is present, respectively denoted by $S_{a b}$ and $S_{0}$. The proposed protocol can be described as follows:

- $S_{0}$ : when $B_{r}=\{\varnothing\}, T_{r}$ keeps on polling both $T_{a}$ and $T_{b}$ until a new "combined" message $W_{c}$ is correctly decoded.

- $S_{a b}$ : when $B_{r}=\left\{W_{c}\right\}$, the relay creates the codeword $\mathbf{x}_{r}\left(W_{c}\right)$ and keeps on broadcasting it until it is correctly received by both terminals.

\section{Throughput}

The throughput of this protocol can still be studied by modeling the system with the Markov chain shown in Fig. 4. Here the relay's buffer can assume only two states $S_{0}$ and $S_{a b}$. However, it is convenient to introduce two further states, $S_{a b^{\prime}}$ and $S_{a b^{\prime \prime}}$, which account for the fact that the relay broadcasts its signal until both users decode the message intended for them. State $S_{a b^{\prime}}\left(S_{a b^{\prime \prime}}\right)$ accounts for the event that terminal 
$T_{a}\left(T_{b}\right)$ has decoded the message $W_{b}\left(W_{a}\right)$ while terminal $T_{b}\left(T_{a}\right)$ has not decoded the message $W_{a}\left(W_{b}\right)$. We define $p_{\text {out }}^{\text {lat }}$ as the probability of the event that the relay does not decode the "combined" message (see below), and $p_{\text {out }, i j}$ is defined as in (7). The indicator variable $\bar{I}_{a}$ is therefore given by $\bar{I}_{a}^{(l a t)}=\left(\pi_{a b}+\pi_{a b^{\prime}}\right)\left(1-p_{\text {out }, r b}\right)$, and similarly for $\bar{I}_{b}$, where $\pi_{a b}$ and $\pi_{a b^{\prime}}$ are the steady-state probabilities of the states $S_{a b}$ and $S_{a b^{\prime}}$ of the Markov chain in Fig. 4. The throughput is therefore given by: $\eta_{\text {lat }}=R_{a} I_{a}^{(l a t)}+R_{b} I_{b}^{(l a t)}$. Using the results in [5], the probability that the relay is not able to decode the sum-codeword is:

$$
\begin{aligned}
& p_{\text {out }}^{\text {lat }}=1-\operatorname{Pr}\left\{R_{a} \leq \log _{2}\left(\frac{P_{a}\left|h_{a r}\right|^{2}}{P_{a}\left|h_{a r}\right|^{2}+P_{b}\left|h_{b r}\right|^{2}}+P_{a}\left|h_{a r}\right|^{2}\right),\right. \\
& \left.R_{b} \leq \log _{2}\left(\frac{P_{b}\left|h_{b r}\right|^{2}}{P_{a}\left|h_{a r}\right|^{2}+P_{b}\left|h_{b r}\right|^{2}}+P_{b}\left|h_{b r}\right|^{2}\right)\right\}
\end{aligned}
$$

\section{NUMERICAL RESUltS}

In this section, we present some numerical results to obtain insight into the performance of the proposed schemes. We focus on the symmetric case $R_{a}=R_{b}=R$ and $P_{a}=$ $P_{b}=P_{r}=P$. Fig. 5 and 6 show the performance of the proposed schemes in terms of normalized throughput $\eta / R$ (packets/slot), focusing on applications with fixed transmission rate, namely $R=0.5,2.5$ and $3.5,8 \mathrm{bps} / \mathrm{Hz}$, respectively. We notice that for low rates (e.g., $R=0.5 \mathrm{bps} / \mathrm{Hz}$ ) the joint decoding (JDF) scheme provides good performance and is not far from the upper bound. However, for increasing value of the transmission rate $R$ the performance of JDF degrades, due to the fact that the joint decoding at the relay becomes critical unless the SNR is not large enough. In contrast, the SDF scheme presents a similar performance gap with respect to the upper bound irrespective of the transmission rate. We can also see that, for large SNR, the throughput of SDF saturates at $\eta / R=2 / 3$. In fact, when transmission becomes essentially error-free, the SDF scheme needs three time slots to perform the bidirectional communication, thus transferring two packets over three time slots. This differs from all other transmission schemes that are able to attain the maximum throughput $\eta / R=1$ for large SNR. For the lattice code-based scheme, for increasing transmission rate $R$, the normalized throughput gets closer to the upper bound thanks to the ability of decoding the sum-codeword instead trying to decode the single messages. However, the lattice scheme provides poor performance in the low-SNR regime. Finally we can see the effect of the noise amplification in the $\mathrm{AF}$ scheme, which generally provides poorer performance with respect to the other schemes.

Fig. 7 shows the throughput $\eta$ for the optimal choice of the transmission rate $R$ versus the SNR $P$ (optimization over $R$ is performed numerically except for the SDF-based scheme as shown in Sec. V-A). It can be seen that the lattice-based strategy outperforms all the other scheme except for the lowSNR regime $(P<10 d B)$. Both DF-based schemes (SDF and JDF) show similar performance for low to moderate-high SNR $(P<30 d B)$, while for very high SNR the SDF is to be preferred to the JDF. Finally, the AF-based scheme shows

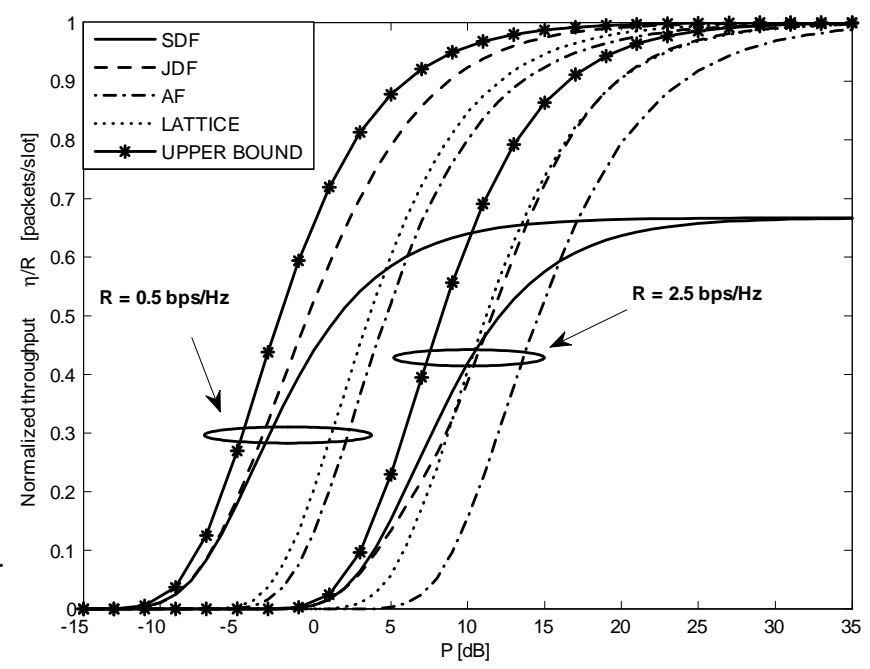

Fig. 5. Normalized throughput $\eta / R$ versus SNR $P$ for a symmetric TWRC $\left(R_{a}=R_{b}=R, P_{a}=P_{b}=P_{r}=P\right.$ ) with $R=0.5$ and $2.5 \mathrm{bps} / \mathrm{Hz}$.

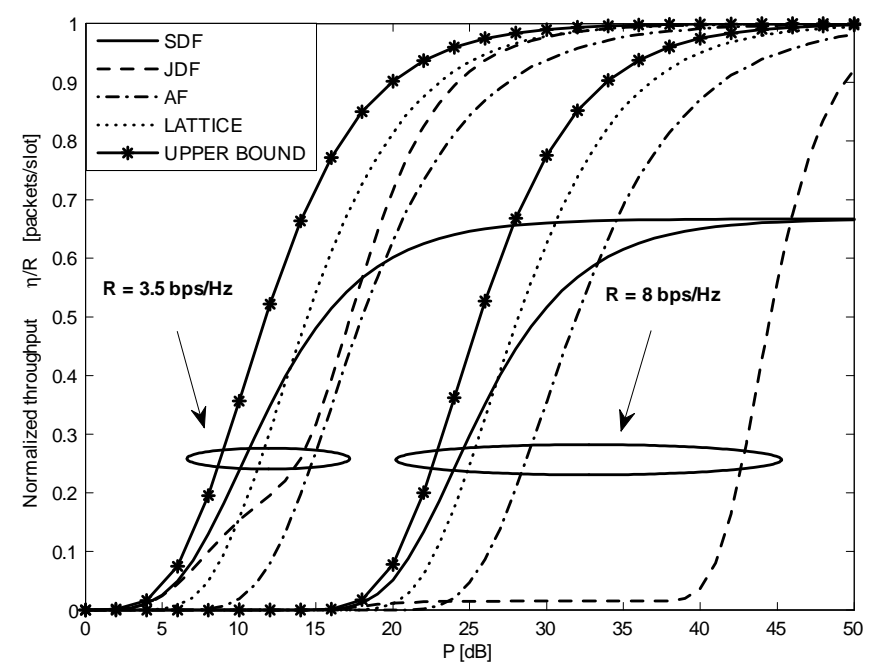

Fig. 6. Normalized throughput $\eta / R$ versus SNR $P$ for a symmetric TWRC $\left(R_{a}=R_{b}=R, P_{a}=P_{b}=P_{r}=P\right.$ ) with $R=3.5$ and $8 \mathrm{bps} / \mathrm{Hz}$.

poorer performance than the DF-based strategies, except in the high-SNR regime $(P>25 d B)$, where it outperforms both DF-based protocols.

\section{CONCLUSION}

A cross layer analysis of physical layer network coding and Type-I HARQ for TWRC over quasi-static fading channels has been proposed. An upper bound on the achievable throughput is derived, and compared to the performance of different schemes with different requirements in term of channel state information. Possible extensions of this work include the analysis of scenarios where the relay has independent data to communicate to the terminals and the investigation of more complex forms of HARQ, such as Chase Combining or Incremental Redundancy (see [6][11] for related work). 


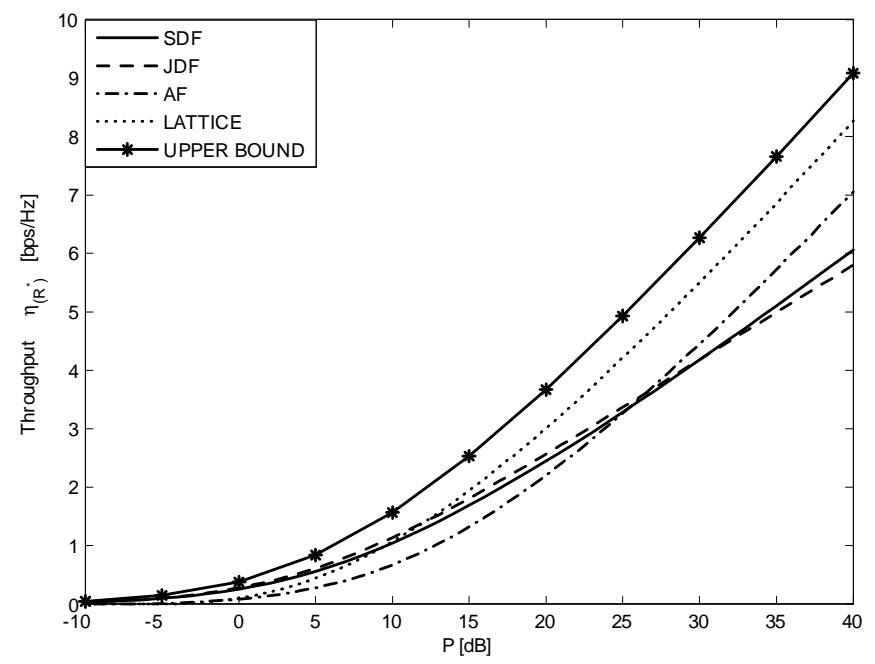

Fig. 7. Throughput $\eta$, maximized over the transmission rate $R$, versus SNR $P$ for a symmetric TWRC $\left(R_{a}=R_{b}=R, P_{a}=P_{b}=P_{r}=P\right)$.

\section{REFERENCES}

[1] P. Popovski and H. Yomo, "Bi-directional amplification of throughput in a wireless multi-hop network," in Proc. VTC 2006, Melbourne, Australia, May 2006.

[2] P. Popovski and H. Yomo, "Physical network coding in two-way wireless relay channels", in Proc. ICC 2007, Glasgow, Scotland, Jun. 2007.

[3] B. Rankov and A. Wittneben, "Spectral efficient signaling for halfduplex relay channels," in Proc. Asilomar Conf. on Signals, Systems and Computers 2005, Pacific Grove, CA, Nov. 2005.

[4] K. Narayanan, M. P. Wilson, and A. Sprintson, "Joint physical layer coding and network coding for bi-directional relaying," in Proc. Allerton Conf. Commun., Control, and Computing, Monticello, IL, Sept. 2007.

[5] W. Nam, S.-Y. Chung and Y.H. Lee, "Capacity bounds for two-way relay channels," in Proc. IEEE Int. Zurich Seminar on Communications, Mar. 2008.

[6] J. K. Sundararajan, D. Shah and M. Medard, "ARQ for network coding," submitted [arXiv:0801.1754v1]

[7] J.N. Laneman, D.N.C. Tse and G.W.Wornell, "Cooperative diversity in wireless networks: efficient protocols and outage behavior," IEEE Trans. Inf. Theory, vol. 50, no. 12, pp. 3062-3080, Dec. 2004.

[8] R. Narasimhan ,"Individual outage rate regions for fading multiple access channels," in Proc. ISIT 2007 , Nice, France, Jun. 2007.

[9] I. Bettesh and S. Shamai, "Optimal power and rate control for minimal average delay: the single-user case," IEEE Trans. Inf. Theory, vol. 52, no.9, pp. 4115-4141, Sep. 2006.

[10] B. Nazer and M. Gastpar, "Compute-and-forward: Harnessing interference with structured codes," in Proc. ISIT 2008, Toronto, Canada, July 2008.

[11] Y. E. Sagduyu and A. Ephremides, "On broadcast stability region in random access through network coding," in Proc. Allerton Conf. Commun., Control, and Computing, Monticello, IL, Sep. 2006. 